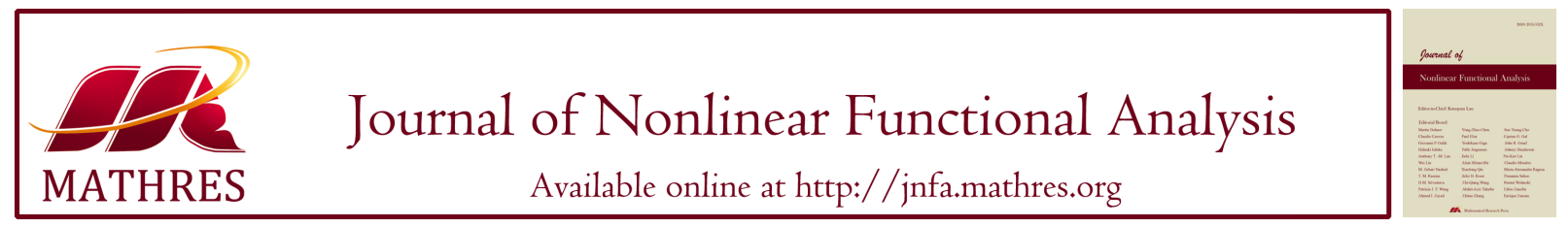

\title{
HOMEOMORPHIC OPTIMALITY CONDITIONS AND DUALITY FOR SEMI-INFINITE PROGRAMMING ON SMOOTH MANIFOLDS
}

\author{
LE THANH TUNG*, DANG HOANG TAM \\ Department of Mathematics, College of Natural Sciences, Can Tho University, Can Tho 900000, Vietnam
}

\begin{abstract}
In this paper, we explore the semi-infinite programming on smooth manifolds. We first discuss the optimality conditions for semi-infinite programming on smooth manifolds via homeomorphic optimality conditions for the associated problems. Further, we present Lagrange, Mond-Weir, and Wolfe type duality for the semi-infinite programming on manifolds, and examine weak and strong duality relations under the $\varphi^{-1}$-convexity assumption.
\end{abstract}

Keywords. Semi-infinite programming; Smooth manifolds; Karush-Kuhn-Tucker optimality conditions; Mond-Weir duality; Wolfe duality.

\section{INTRODUCTION}

The semi-infinite programming problems in Euclidean spaces are optimization problems with the infinite number of constraints. Due to their important meanings in theoretical aspect and practical applications, these problems have been recently studied in many papers, see e.g., [1, $2,3,4,5,6,7,8,9,10,11]$ and the references therein.

To deal with some optimization problems in non-Euclidean spaces, such as, learning algorithms for neural networks [12], image and video-based recognition [13], and variational control problems [14], the study of optimization on manifolds have been investigated extensively in recent years. In [15], the weak sharp minima for constrained optimization problems on Riemannian manifolds and Hadamard manifolds were discussed. The first-order and the secondorder optimality conditions for nonlinear programming problem on Riemannian manifolds were studied via the Clarke generalized gradient in [16]. The paper [17] established both necessary and sufficient optimality conditions for vector equilibrium problems on Hadamard manifolds. Intrinsic formulation of Karush-Kuhn-Tucker (KKT) necessary optimality conditions were given in [18]. In [19], the KKT sufficient optimality conditions for optimization problem with interval-valued objective function on Hadamard manifolds were considered. However, to

${ }^{*}$ Correspoinding author.

E-mail address: lttung@ctu.edu.vn (L.T. Tung).

Received December 12, 2020; Accepted April 28, 2021.

(C)2021 Journal of Nonlinear Functional Analysis 
the best of our knowledge, there is no paper dealing with the optimality conditions and duality for semi-infinite programming on smooth manifolds.

Motivated by the above observations, in this paper, we establish Karush-Kuhn-Tucker optimality conditions and investigate duality problems for semi-infinite programming on smooth manifolds. The organization of the paper is as follows. Section 2 recalls basic notions and presents the $\varphi^{-1}$-convexity notion. Karush-Kuhn-Tucker necessary and sufficient optimality conditions for the semi-infinite programming on smooth manifolds are discussed in Section 3. Section 4 is concerned with exploring Lagrange, Mond-Weir and Wolfe dual problems of semiinfinite programming on smooth manifold. The results of the paper are illuminated by several examples.

\section{PRELIMINARIES}

In this paper, the notation $\langle\cdot, \cdot\rangle$ is utilized to denote the inner product in the Euclidean space $\mathscr{E}$. For a given $\bar{x} \in \mathscr{E}, \mathscr{U}(\bar{x})$ is the system of the open neighborhoods of $\bar{x}$. For $A \subseteq \mathscr{E}, \operatorname{int} A, \operatorname{cl} A$, $\operatorname{aff} A, \operatorname{span} A$, and $\operatorname{co} A$ stand for its interior, closure, affine hull, linear hull, and convex hull of $A$, respectively (shortly, resp). The cone and the convex cone (containing the origin) generated by $A$ are indicated, resp, by $\operatorname{cone} A, \operatorname{pos} A$. The negative polar cone, and the strictly negative polar cone (see, e.g., $[11,20,21])$ of $A$ are defined, resp, by

$$
A^{-}:=\left\{x^{*} \in \mathscr{E} \mid\left\langle x^{*}, x\right\rangle \leq 0, \forall x \in A\right\},
$$

and

$$
A^{s}:=\left\{x^{*} \in \mathscr{E} \mid\left\langle x^{*}, x\right\rangle<0, \forall x \in A\right\} .
$$

We can check that if $A^{s} \neq \emptyset$, then $\operatorname{cl} A^{s}=A^{-}$. Indeed, let $x^{*} \in \operatorname{cl} A^{s}$. Then, there exists a sequence $x_{k}^{*} \rightarrow x^{*}$ satisfying $\left\langle x_{k}^{*}, x\right\rangle<0$ for all $x \in A$. Letting $k$ to infinity, one has $\left\langle x^{*}, x\right\rangle \leq 0$ for all $x \in A$, which leads to $x^{*} \in A^{-}$. Conversely, let $x^{*} \in A^{-}$. Then, $\left\langle x^{*}, x\right\rangle \leq 0$ for all $x \in A$. We deduce from $A^{s} \neq \emptyset$ that there is $\bar{x}^{*} \in A^{s}$ such that $\left\langle\bar{x}^{*}, x\right\rangle<0$ for all $x \in A$. Setting $x_{k}^{*}=x^{*}+\frac{1}{k} \bar{x}^{*}$, we get $x_{k}^{*} \rightarrow x^{*}$ and $x_{k}^{*} \in A^{s}$ due to

$$
\left\langle x_{k}^{*}, x\right\rangle=\left\langle x^{*}, x\right\rangle+\frac{1}{k}\left\langle\bar{x}^{*}, x\right\rangle<0 \forall x \in A,
$$

i.e., $x^{*} \in \operatorname{cl} A^{s}$.

Moreover, the bipolar theorem (see, e.g., $[20,22]$ ) tells us that $A^{--}=\mathrm{cl}$ cone $A$. For a given nonempty subset $A$ of $\mathscr{E}$, the contingent cone [20,22] of $A$ at $\bar{x} \in \operatorname{cl} A$ is

$$
\mathscr{T}(A, \bar{x}):=\left\{x \in \mathscr{E} \mid \exists \tau_{k} \downarrow 0, \exists x_{k} \rightarrow x, \forall k \in \mathbb{N}, \bar{x}+\tau_{k} x_{k} \in A\right\} .
$$

Notice that if $A$ is a convex set, then $\mathscr{T}(A, \bar{x})=\operatorname{clcone}(A-\bar{x})$. If $\left\langle x^{*}, x\right\rangle \geq 0$ for all $x^{*} \in A^{*}$, where $A^{*}$ is a subset of the dual space of $\mathscr{E}$, we write $\left\langle A^{*}, x\right\rangle \geq 0$. The cardinality of the index set $I$ is denoted by $|I|$. The map $\varphi$ from an Euclidean space $\mathscr{E}_{1}$ to another Euclidean space $\mathscr{E}_{2}$ is said to be of class $C^{1}\left(C^{\infty}\right.$, resp) if $\varphi$ is continuously differentiable (infinitely continuously differentiable, resp) on $\mathscr{E}_{1}$. Recall the following definition of the smooth manifolds; see [23, 24, $25,26,27,28]$ for more details.

Definition 2.1. Let $\mathscr{E}$ be an Euclidean space, and let $M^{n} \subseteq \mathscr{E}$ be a Hausdorff (induced) topological space with a countable base. $M^{n}$ is said to be endowed with a smooth structure of dimension $n$ if there exists an index set $I$, a collection of (topological) open subsets $\left\{U_{\alpha}\right\}_{\alpha \in I}$ covering $M^{n}$, together with a collection of homeomorphisms $\varphi_{\alpha}: U_{\alpha} \rightarrow \varphi_{\alpha}\left(U_{\alpha}\right) \subseteq \mathbb{R}^{n}$ such that the transition 
$\operatorname{map} \varphi_{\beta} \circ \varphi_{\alpha}^{-1}: \varphi_{\alpha}\left(U_{\alpha} \cap U_{\beta}\right) \rightarrow \varphi_{\beta}\left(U_{\alpha} \cap U_{\beta}\right)$ are of class $C^{\infty}$ for all $\alpha, \beta \in I$. A pair $\left(U_{\alpha}, \varphi_{\alpha}\right)$ is called a smooth chart, and the maximal collection $\mathscr{A}:=\left\{U_{\alpha}\right\}_{\alpha \in I}$ is said to be a smooth atlas. Then, the pair $\left(M^{n}, \mathscr{A}\right)$ is called a smooth manifold of dimension $n$.

The function $h: M^{n} \rightarrow \mathbb{R}$ is assumed to be $C^{1}$-property if $h \circ \varphi_{\alpha}^{-1}: \varphi_{\alpha}\left(U_{\alpha}\right) \rightarrow \mathbb{R}$ is of class $C^{1}$ for every chart $\left(U_{\alpha}, \varphi_{\alpha}\right)$ in the smooth atlas $\mathscr{A}$. For an open set $X \subset M^{n}$, the function $h: X \subset M^{n} \rightarrow \mathbb{R}$ has the $C^{1}$-property on $X$ if $h \circ \varphi_{\alpha}^{-1}: \varphi_{\alpha}\left(U_{\alpha} \cap X\right) \rightarrow \mathbb{R}$ is of class $C^{1}$ for every chart $\left(U_{\alpha}, \varphi_{\alpha}\right)$. It is well-known that $\mathbb{R}^{n}$, spheres $\mathbb{S}^{n}$, tori, $G L(n), S O(n)$, Grassmannian manifold, and Stiefel manifold are examples of smooth manifolds.

Definition 2.2. Let $(U, \varphi)$ be a chart of $M$ about $\bar{x}$, and let $h \circ \varphi^{-1}: \varphi(U) \subset \mathbb{R}^{n} \rightarrow \mathbb{R}$ be a continuously differentiable function on $\varphi(U)$. The map $h$ is said to be $\varphi^{-1}$-convex at $\bar{z}=\varphi(\bar{x})$ on $\varphi(U)$ iff

$$
h\left(\varphi^{-1}(z)\right)-h\left(\varphi^{-1}(\bar{z})\right) \geq\left\langle\nabla\left(h \circ \varphi^{-1}\right)(\bar{z}), z-\bar{z}\right\rangle, \forall z \in \varphi(U),
$$

and, $h$ is said to be $\varphi^{-1}$-convex on $\varphi(U)$ if $h$ is $\varphi^{-1}$-convex at each $z \in \varphi(U)$.

Example 2.3. Let $\mathscr{E}=\mathbb{R}^{2}, M=\operatorname{int} \mathbb{R}_{+}^{2}=\left\{x=\left(x_{1}, x_{2}\right) \in \mathbb{R}^{2} \mid x_{1}>0, x_{2}>0\right\}$, and let $\varphi: U=$ $M \rightarrow \varphi(U) \subseteq \mathbb{R}^{2}$ be defined by $\varphi(x)=\left(\sqrt[3]{x_{1}}, \ln \left(x_{2}\right)\right)$. By some calculations, we get that

$$
\varphi^{-1}(z)=\left(z_{1}^{3}, e^{z_{2}}\right), \varphi(U)=\left\{x=\left(x_{1}, x_{2}\right) \in \mathbb{R}^{2} \mid x_{1}>0\right\}
$$

is an open in $\mathbb{R}^{2}$, which in turn implies that $\varphi$ is a homeomorphism, and $M$ is a smooth manifold with the single chart $(M, \varphi)$. Let $h: M \rightarrow \mathbb{R}$ be defined by $h(x)=\sqrt[3]{x_{1}}+\ln \left(x_{2}\right)$. Then, we can check that $h$ is not convex on $M$ in the usual sense. However, one has

$$
\left(h \circ \varphi^{-1}\right)(z)=h\left(\varphi^{-1}(z)\right)=h\left(z_{1}^{3}, e^{z_{2}}\right)=z_{1}+z_{2},
$$

leading that $h$ is $\varphi^{-1}$-convex on $\varphi(M)$.

Remark 2.4. The Definition 2.2 employs some ideas of the $E$-convexity, see [29, 30, 31, 32, 33]. However, the $\varphi^{-1}$-convexity is different from the $E$-convexity in general.

Lemma 2.5. [34] Let $\left\{C_{t} \mid t \in \Gamma\right\}$ be an arbitrary collection of nonempty convex sets in $\mathbb{R}^{n}$, and let $K:=\operatorname{pos}\left(\bigcup_{t \in \Gamma} C_{t}\right)$. Then, every nonzero vector of $K$ can be expressed as a non-negative linear combination of $n$ or fewer linear independent vectors, each belonging to a different $C_{t}$.

Lemma 2.6. [35] Suppose that $S, P$ are arbitrary (possibly infinite) index sets, and $a_{s}=a(s)=$ $\left(a_{1}(s), \ldots, a_{n}(s)\right)$ maps $S$ onto $\mathbb{R}^{n}$, and so does $a_{p}$. Suppose that the set $\operatorname{co}\left\{a_{s}, s \in S\right\}+\operatorname{pos}\left\{a_{p}, p \in\right.$ $P\}$ is closed. Then the following statements are equivalent:

$$
I: \quad\left\{\begin{array}{l}
\left\langle a_{s}, x\right\rangle<0, s \in S, S \neq \emptyset \\
\left\langle a_{p}, x\right\rangle \leq 0, p \in P
\end{array} \quad \text { has no solution } x \in \mathbb{R}^{n} ;\right.
$$

Lemma 2.7. [21] If $X$ is a nonempty compact subset of $\mathbb{R}^{n}$, then

(i) $\operatorname{co} X$ is a compact set;

(ii) If $0 \notin \operatorname{co} X$, then $\operatorname{pos} X$ is a closed cone. 


\section{KKT OPTIMALITY CONDITIONS}

In this paper, we consider the following semi-infinite programming:

$$
\begin{aligned}
& (P): \min f(x) \\
& \text { s.t. } g_{t}(x) \leq 0, t \in T, \\
& x \in M^{n},
\end{aligned}
$$

where $f, g_{t}(t \in T)$ are functions from $\mathscr{E}$ to $\mathbb{R}$. The constraint $x \in M^{n}$ means that $f, g_{t}(t \in T)$ are defined on the manifold $M^{n}$, i.e., $f, g_{t}: M^{n} \subset \mathscr{E} \rightarrow \mathbb{R}$; see [16, 18]. The index set $T$ is an arbitrary nonempty set, not necessary finite. A point $\bar{x}$ is a locally optimal solution of $(P)$ if there exists a neighborhood $U_{x} \subseteq \mathscr{E}$ of $\bar{x}$ such that

$$
f(x) \geq f(\bar{x}), \forall x \in \Omega \cap U_{x},
$$

where $\Omega:=\left\{x \in M^{n} \mid g_{t}(x) \leq 0, t \in T\right\}$ is the feasible solution set of $(P)$. If $U_{x}=\mathscr{E}$, the word "locally" is omitted. Since $\Omega \subset M^{n}$, the word "locally" could also be omitted in the case that $U_{x}=\mathscr{U}$, where $\mathscr{U}$ is an open set in $\mathscr{E}$ containing $M^{n}$.

For an arbitrary chart $(U, \varphi)$, the semi-infinite programming with respect to $\varphi$ is

$$
\begin{aligned}
\left(P_{\varphi}\right): & \min f(x) \\
\text { s.t. } & g_{t}(x) \leq 0, t \in T, \\
& x \in U .
\end{aligned}
$$

Denote by $\Omega_{\varphi}:=\left\{x \in U \mid g_{t}(x) \leq 0, t \in T\right\}$ the feasible solution set of $\left(P_{\varphi}\right)$. A point $\bar{x}$ is a locally optimal solution of $\left(P_{\varphi}\right)$ if there exists a neighborhood $U_{x} \subseteq \mathscr{E}$ of $\bar{x}$ such that

$$
f(x) \geq f(\bar{x}), \forall x \in \Omega_{\varphi} \cap U_{x} .
$$

If $U_{x}=\mathscr{U}$, where $\mathscr{U}$ is an open set in $\mathscr{E}$ containing $U$, the word "locally" is omitted. If the atlas of $M^{n}$ consists of the single chart $\mathscr{A}=\{(U, \varphi)\}$ with $U=M^{n}$, then $\left(P_{\varphi}\right)$ coincides with $(P)$.

For an arbitrary chart $(U, \varphi)$, let us consider the associated $\varphi^{-1}$-semi-infinite programming $\left(P_{\varphi^{-1}}\right)$ of $\left(P_{\varphi}\right)$ as follows:

$$
\begin{aligned}
&\left(P_{\varphi^{-1}}\right): \min \left(f \circ \varphi^{-1}\right)(z) \\
& \text { s.t. } \quad\left(g_{t} \circ \varphi^{-1}\right)(z) \leq 0, \quad t \in T, \\
& z \in \varphi(U),
\end{aligned}
$$

where $f \circ \varphi^{-1}, g_{t} \circ \varphi^{-1}(t \in T)$ are functions from an open set $\varphi(U)$ of $\mathbb{R}^{n}$ to $\mathbb{R}$. Denote by $\Omega_{\varphi^{-1}}:=\left\{z \in \varphi(U) \mid\left(g_{t} \circ \varphi^{-1}\right)(z) \leq 0, t \in T\right\}$ the feasible solution set of $\left(P_{\varphi^{-1}}\right)$ and the index set of all active constraints at $\bar{z} \in \Omega_{\varphi^{-1}}$ is $T_{\varphi^{-1}}(\bar{z}):=\left\{t \in T \mid\left(g_{t} \circ \varphi^{-1}\right)(\bar{z})=0\right\}$. The set of active constraint multipliers at $\bar{z} \in \Omega_{\varphi^{-1}}$ is $\Lambda_{\varphi^{-1}}(\bar{z}):=\left\{\lambda \in \mathbb{R}_{+}^{|T|} \mid \lambda_{t}\left(g_{t} \circ \varphi^{-1}\right)(\bar{z})=0, \forall t \in T\right\}$. Notice that $\lambda \in \Lambda_{\varphi^{-1}}(\bar{z})$ if there exists a finite index set $J \subset T_{\varphi^{-1}}(\bar{z})$ such that $\lambda_{t}>0$ for all $t \in J$ and $\lambda_{t}=0$ for all $t \in T_{\varphi^{-1}}(\bar{z}) \backslash J$.

Lemma 3.1. For an arbitrary chart $(U, \varphi), \varphi\left(\Omega_{\varphi}\right)=\Omega_{\varphi^{-1}}$ and $\varphi^{-1}\left(\Omega_{\varphi^{-1}}\right)=\Omega_{\varphi}$.

Proof. Since

$$
\begin{aligned}
x \in \Omega_{\varphi} & \Leftrightarrow x \in U, g_{t}(x) \leq 0, \forall t \in T \\
& \Leftrightarrow \varphi(x) \in \varphi(U),\left(g_{t} \circ \varphi^{-1}\right)(\varphi(x)) \leq 0, \forall t \in T \\
& \Leftrightarrow z=\varphi(x) \in \Omega_{\varphi^{-1}} \\
& \Leftrightarrow x=\varphi^{-1}(z) \in \varphi^{-1}\left(\Omega_{\varphi^{-1}}\right),
\end{aligned}
$$


we conclude the desire conclusion immediately.

Definition 3.2. Let $(U, \varphi)$ be an arbitrary chart. A point $\bar{z} \in \Omega_{\varphi^{-1}}$ is a locally optimal solution of $\left(P_{\varphi^{-1}}\right)$ if there exists a neighborhood $U_{z} \subseteq \mathbb{R}^{n}$ of $\bar{z}$ such that

$$
\left(f \circ \varphi^{-1}\right)(z) \geq\left(f \circ \varphi^{-1}\right)(\bar{z}), \forall z \in \Omega_{\varphi^{-1}} \cap U_{z} .
$$

If $U_{z}=\mathbb{R}^{n}$, the word "locally" is dropped. Since $\varphi(U)$ is an open set in $\mathbb{R}^{n}$, we can drop the word "locally" if $U_{z}=\varphi(U)$.

Lemma 3.3. Let $\left(M^{n}, \mathscr{A}\right)$ be a smooth manifold.

(i) Assume that $(U, \varphi)$ is an arbitrary chart in $\mathscr{A}$. A point $\bar{x} \in \Omega_{\varphi}$ is a (locally) optimal solution of $\left(P_{\varphi}\right)$ if and only if $\bar{z}=\varphi(\bar{x})$ is a (locally) optimal solution of $\left(P_{\varphi^{-1}}\right)$.

(ii) A point $\bar{x} \in \Omega$ is a locally optimal solution of $(P)$ if and only if $\varphi(\bar{x})$ is a locally optimal solution of $\left(P_{\varphi^{-1}}\right)$ for any chart $(U, \varphi)$ about $\bar{x}$.

(iii) If the atlas $\mathscr{A}$ of $M^{n}$ consists of the finite charts, i.e. $\mathscr{A}=\left\{\left(U_{1}, \varphi_{1}\right), \ldots,\left(U_{p}, \varphi_{p}\right)\right\}$, and $\bar{x}^{i}$ is optimal solutions of $\left(P_{\varphi_{i}}\right)$ for $i=1, \ldots, p$, then $\bar{x}$ satisfying $f(\bar{x})=\min _{i=1, \ldots, p}\left\{f\left(\bar{x}^{i}\right)\right\}$ is an optimal solution of $(P)$.

Proof. (i) Let $\bar{x} \in \Omega_{\varphi}$ be a (locally) optimal solution of $\left(P_{\varphi}\right)$. Then, there exists a neighborhood $U_{x}$ in $\mathscr{E}$ satisfying $U_{x} \cap \Omega_{\varphi} \subseteq U$, by shrinking if necessary, such that

$$
f(x) \geq f(\bar{x}), \forall x \in U_{x} \cap \Omega_{\varphi} .
$$

Since $\varphi\left(U_{x}\right)$ is an open neighborhood of $\varphi(\bar{x})$ and $\varphi\left(\Omega_{\varphi}\right)=\Omega_{\varphi^{-1}}$, the above inequality implies that

$$
\left(f \circ \varphi^{-1}\right)(\varphi(x)) \geq\left(f \circ \varphi^{-1}\right)(\varphi(\bar{x})), \forall \varphi(x) \in \varphi\left(U_{x}\right) \cap \Omega_{\varphi^{-1}} .
$$

Hence, $\varphi(\bar{x})$ is a (locally) optimal solution of $\left(P_{\varphi^{-1}}\right)$.

Conversely, let $\bar{z}=\varphi(\bar{x}) \in \Omega_{\varphi^{-1}}$ be a (locally) optimal solution of $\left(P_{\varphi^{-1}}\right)$. Then, there exists a neighborhood $U_{z} \subseteq \varphi(U)$ in $\mathbb{R}^{n}$ such that

$$
\left(f \circ \varphi^{-1}\right)(z) \geq\left(f \circ \varphi^{-1}\right)(\varphi(\bar{x})), \forall z \in U_{z} \cap \Omega_{\varphi^{-1}} .
$$

Since $\varphi^{-1}\left(U_{z}\right)$ is an open neighborhood of $\varphi^{-1}(\varphi(\bar{x}))=\bar{x}$ in $\mathscr{E}$ and $\varphi^{-1}\left(\Omega_{\varphi^{-1}}\right)=\Omega_{\varphi}$, the above inequality implies that

$$
f\left(\varphi^{-1}(z)\right) \geq f(\bar{x}), \forall \varphi^{-1}(z) \in \varphi^{-1}\left(U_{z}\right) \cap \Omega_{\varphi} .
$$

Therefore, $\bar{x}$ is a (locally) optimal solution of $\left(P_{\varphi}\right)$.

(ii) Let $\bar{x} \in \Omega$ be a locally optimal solution of $(P)$, and let $(U, \varphi)$ be an arbitrary chart about $\bar{x}$. Then, there exists a neighborhood $U_{x}$ in $\mathscr{E}$ satisfying $U_{x} \cap \Omega_{\varphi} \subseteq U$ such that

$$
f(x) \geq f(\bar{x}), \forall x \in U_{x} \cap \Omega_{\varphi} .
$$

Since $\varphi\left(U_{x}\right)$ is an open neighborhood of $\varphi(\bar{x})$ and $\varphi\left(\Omega_{\varphi}\right)=\Omega_{\varphi^{-1}}$, the above inequality derives that

$$
\left(f \circ \varphi^{-1}\right)(\varphi(x)) \geq\left(f \circ \varphi^{-1}\right)(\varphi(\bar{x})), \forall \varphi(x) \in \varphi\left(U_{x}\right) \cap \Omega_{\varphi^{-1}} .
$$

Hence, $\varphi(\bar{x})$ is a locally optimal solution of $\left(P_{\varphi^{-1}}\right)$. 
Conversely, let $(U, \varphi)$ be an arbitrary chart about $\bar{x}$, and let $\bar{z}=\varphi(\bar{x}) \in \Omega_{\varphi^{-1}}$ be a locally optimal solution of $\left(P_{\varphi^{-1}}\right)$. Then, there exists a neighborhood $U_{z} \subseteq \varphi(U)$, by contracting if necessary, such that

$$
\left(f \circ \varphi^{-1}\right)(z) \geq\left(f \circ \varphi^{-1}\right)(\varphi(\bar{x})), \forall z \in U_{z} \cap \Omega_{\varphi^{-1}} .
$$

As $\varphi^{-1}\left(U_{z}\right)$ is an open neighborhood of $\varphi^{-1}(\varphi(\bar{x}))=\bar{x}$, and $\varphi^{-1}\left(\Omega_{\varphi^{-1}}\right)=\Omega_{\varphi}$, the above leads that

$$
f\left(\varphi^{-1}(z)\right) \geq f(\bar{x}), \forall \varphi^{-1}(z) \in \varphi^{-1}\left(U_{z}\right) \cap \Omega_{\varphi} \subseteq \varphi^{-1}\left(U_{z}\right) \cap \Omega .
$$

So, $\bar{x}$ is a locally optimal solution of $(P)$.

(iii) The conclusion could be derived directly from the definition.

Example 3.4. Let $\mathscr{E}=\mathbb{R}^{2}$, and let $M^{1}=\mathbb{S}^{1}=\left\{x \in \mathbb{R}^{2} \mid x_{1}^{2}+x_{2}^{2}=1\right\}$. Then, $M^{1}$ is a smooth manifold with the atlas $\mathscr{A}=\left\{\left(U_{i}, \varphi_{i}\right), i=1, \ldots, 4\right\}$, where

$$
\begin{gathered}
U_{1}=\left\{x \in \mathbb{S}^{1} \mid x_{1}>0\right\}, \varphi_{1}\left(x_{1}, x_{2}\right)=x_{2}, \varphi_{1}^{-1}(z)=\left(\sqrt{1-z^{2}}, z\right), \\
U_{2}=\left\{x \in \mathbb{S}^{1} \mid x_{1}<0\right\}, \varphi_{2}\left(x_{1}, x_{2}\right)=x_{2}, \varphi_{2}^{-1}(z)=\left(-\sqrt{1-z^{2}}, z\right), \\
U_{3}=\left\{x \in \mathbb{S}^{1} \mid x_{2}>0\right\}, \varphi_{3}\left(x_{1}, x_{2}\right)=x_{1}, \varphi_{3}^{-1}(z)=\left(z, \sqrt{1-z^{2}}\right),
\end{gathered}
$$

and

$$
U_{4}=\left\{x \in \mathbb{S}^{1} \mid x_{2}<0\right\}, \varphi_{4}\left(x_{1}, x_{2}\right)=x_{1}, \varphi_{4}^{-1}(z)=\left(z,-\sqrt{1-z^{2}}\right) .
$$

Consider the problem

$$
\begin{aligned}
& (P): \min f(x)=x_{2}^{2} \\
& \text { s.t. } \quad g_{t}(x)=-x_{2}+t \leq 0, t \in T=\left[0, \frac{1}{2}\right], \\
& \quad x \in \mathbb{S}^{1}=\left\{x \in \mathbb{R}^{2} \mid x_{1}^{2}+x_{2}^{2}=1\right\} .
\end{aligned}
$$

Then, $\Omega=\left\{x \in \mathbb{S}^{1} \mid x_{2} \geq \frac{1}{2}\right\}$ and $\bar{x}=\left( \pm \frac{\sqrt{3}}{2}, \frac{1}{2}\right)$ is an optimal solutions of $(P)$. Since $U_{4} \cap \Omega=$ $\emptyset$, we consider only 3 semi-infinite programming problems with respect to $\varphi_{i}(i=1,2,3)$ as follows.

$*\left(P_{\varphi_{1}}\right): \min _{x \in U_{1}}\left\{f(x)=x_{2}^{2} \mid g_{t}(x)=-x_{2}+t \leq 0, t \in T\right\}, \Omega_{\varphi_{1}}=\left\{x \in U_{1} \mid x_{2} \geq \frac{1}{2}\right\}$ and $\bar{x}=\left(\frac{\sqrt{3}}{2}, \frac{1}{2}\right)$ is an optimal solution of $\left(P_{\varphi_{1}}\right)$.

$\left(P_{\varphi_{1}^{-1}}\right): \min _{z \in \varphi_{1}\left(U_{1}\right)}\left\{\left(f \circ \varphi_{1}^{-1}\right)(z)=z^{2} \mid\left(g_{t} \circ \varphi_{1}^{-1}\right)(z)=-z+t \leq 0, t \in T\right\}, \Omega_{\varphi_{1}^{-1}}=\{z \in(-1,1) \mid$ $\left.z \geq \frac{1}{2}\right\}$ and $\bar{z}=\frac{1}{2}$ is an optimal solution of $\left(P_{\varphi_{1}^{-1}}\right)$.

$*\left(P_{\varphi_{2}}\right): \min _{x \in U_{2}}\left\{f(x)=x_{2}^{2} \mid g_{t}(x)=-x_{2}+t \leq 0, t \in T\right\}, \Omega_{\varphi_{2}}=\left\{x \in U_{2} \mid x_{2} \geq \frac{1}{2}\right\}$ and $\bar{x}=\left(-\frac{\sqrt{3}}{2}, \frac{1}{2}\right)$ is an optimal solution of $\left(P_{\varphi_{2}}\right)$.

$\left(P_{\varphi_{2}^{-1}}\right): \min _{z \in \varphi_{2}\left(U_{2}\right)}\left\{\left(f \circ \varphi_{2}^{-1}\right)(z)=z^{2} \mid\left(g_{t} \circ \varphi_{2}^{-1}\right)(z)=-z+t \leq 0, t \in T\right\}, \Omega_{\varphi_{2}^{-1}}=\{z \in(-1,1) \mid$ $\left.z \geq \frac{1}{2}\right\}$ and $\bar{z}=\frac{1}{2}$ is an optimal solution of $\left(P_{\varphi_{2}^{-1}}\right)$.

$*\left(P_{\varphi_{3}}\right): \min _{x \in U_{3}}\left\{f(x)=x_{2}^{2} \mid g_{t}(x)=-x_{2}+t \leq 0, t \in T\right\}, \Omega_{\varphi_{3}}=\left\{x \in U_{3} \mid x_{2} \geq \frac{1}{2}\right\}$ and $\bar{x}=\left( \pm \frac{\sqrt{3}}{2}, \frac{1}{2}\right)$ are optimal solutions of $\left(P_{\varphi_{3}}\right)$.

$\left(P_{\varphi_{3}^{-1}}\right): \min _{z \in \varphi_{3}\left(U_{3}\right)}\left\{\left(f \circ \varphi_{3}^{-1}\right)(z)=1-z^{2} \mid\left(g_{t} \circ \varphi_{3}^{-1}\right)(z)=-\sqrt{1-z^{2}}+t \leq 0, t \in T\right\}, \Omega_{\varphi_{3}^{-1}}=\{z \in$ $\left.(-1,1) \mid \sqrt{1-z^{2}} \geq \frac{1}{2}\right\}$ and $\bar{z}= \pm \frac{\sqrt{3}}{2}$ are optimal solutions of $\left(P_{\varphi_{3}^{-1}}\right)$.

So, the conclusion of Lemma 3.3 holds. 
In the sequel, we always assume that $f, g_{t}(t \in T)$ are of $C^{1}$-property on $M^{n}$.

Definition 3.5. Let $(U, \varphi)$ be an arbitrary chart. The $\left(A C Q_{\varphi^{-1}}\right)$ holds at $\bar{z} \in \Omega_{\varphi^{-1}}$ if

$$
\left(\bigcup_{t \in T_{\varphi^{-1}}(\bar{z})} \nabla\left(g_{t} \circ \varphi^{-1}\right)(\bar{z})\right)^{-} \subseteq \mathscr{T}\left(\Omega_{\varphi^{-1}}, \bar{z}\right),
$$

and the set $\Delta_{\varphi^{-1}}:=\operatorname{pos} \underset{t \in T_{\varphi^{-1}}(\bar{z})}{\bigcup} \nabla\left(g_{t} \circ \varphi^{-1}\right)(\bar{z})$ is closed.

Proposition 3.6. Let $(U, \varphi)$ be an arbitrary chart. Suppose that $\bar{z}$ is a locally optimal solution of $\left(P_{\varphi^{-1}}\right)$, and $\left(A C Q_{\varphi^{-1}}\right)$ holds at $\bar{z}$. Then, there exists $\lambda \in \Lambda_{\varphi^{-1}}(\bar{z})$ such that

$$
\nabla\left(f \circ \varphi^{-1}\right)(\bar{z})+\sum_{t \in T} \lambda_{t} \nabla\left(g_{t} \circ \varphi^{-1}\right)(\bar{z})=0 .
$$

Proof. We first justify that

$$
\left(\nabla\left(f \circ \varphi^{-1}\right)(\bar{z})\right)^{s} \cap \mathscr{T}\left(\Omega_{\varphi^{-1}}, \bar{z}\right)=\emptyset .
$$

* Case 1. $\nabla\left(f \circ \varphi^{-1}\right)(\bar{z})=0$. Then, $\left(\nabla\left(f \circ \varphi^{-1}\right)(\bar{z})\right)^{s}=\emptyset$. Hence, (3.1) holds for the Case 1 . * Case 2. $\nabla\left(f \circ \varphi^{-1}\right)(\bar{z}) \neq 0$. Then, suppose to the contrary that there exists $d \in(\nabla(f \circ$ $\left.\left.\varphi^{-1}\right)(\bar{z})\right)^{s} \cap \mathscr{T}\left(\Omega_{\varphi^{-1}}, \bar{z}\right)$. Hence, one has $\left\langle\nabla\left(f \circ \varphi^{-1}\right)(\bar{z}), d\right\rangle<0$. Since $d \in \mathscr{T}\left(\Omega_{\varphi^{-1}}, \bar{z}\right)$, there exist $\tau_{k} \downarrow 0$ and $d_{k} \rightarrow d$ such that $\bar{z}+\tau_{k} d_{k} \in \Omega_{\varphi^{-1}}$ for all $k$. We derive from $f$ is of $C^{1}$-property at $\bar{z}$ that

$$
\left(f \circ \varphi^{-1}\right)\left(\bar{z}+\tau_{k} d_{k}\right)=\left(f \circ \varphi^{-1}\right)(\bar{z})+\tau_{k}\left\langle\nabla\left(f \circ \varphi^{-1}\right)(\bar{z}), d_{k}\right\rangle+o\left(\left\|\tau_{k} d_{k}\right\|\right) .
$$

Consequently,

$$
\begin{gathered}
\frac{\left(f \circ \varphi^{-1}\right)\left(\bar{z}+\tau_{k} d_{k}\right)-\left(f \circ \varphi^{-1}\right)(\bar{z})}{\tau_{k}}=\left\langle\nabla\left(f \circ \varphi^{-1}\right)(\bar{z}), d_{k}\right\rangle+\frac{o\left(\left\|\tau_{k} d_{k}\right\|\right)}{\tau_{k}} \\
\underset{k \rightarrow \infty}{\longrightarrow}\left\langle\nabla\left(f \circ \varphi^{-1}\right)(\bar{z}), d\right\rangle<0 .
\end{gathered}
$$

Thus, we affirm the existence of $k>\bar{k}$ large enough such that $\bar{z}+\tau_{k} d_{k} \in \Omega_{\varphi^{-1}}$ and

$$
\left(f \circ \varphi^{-1}\right)\left(\bar{z}+\tau_{k} d_{k}\right)<\left(f \circ \varphi^{-1}\right)(\bar{z})
$$

which contradicts the fact that $\bar{z}$ is a locally optimal solution of $\left(P_{\varphi^{-1}}\right)$. Hence, claim (3.1) holds for the Case 2, which leads to the fact that (3.1) satisfies both two possibilities.

We derive from $(3.1)$ and $\left(A C Q_{\varphi^{-1}}\right)$ that

$$
\left(\nabla\left(f \circ \varphi^{-1}\right)(\bar{z})\right)^{s} \cap\left(\bigcup_{t \in T_{\varphi^{-1}}(\bar{z})} \nabla\left(g_{t} \circ \varphi^{-1}\right)(\bar{z})\right)^{-} \subseteq\left(\nabla\left(f \circ \varphi^{-1}\right)(\bar{z})\right)^{s} \cap \mathscr{T}\left(\Omega_{\varphi^{-1}}, \bar{z}\right)=\emptyset .
$$

This leads that there is no $d \in \mathbb{R}^{n}$ fulfilling

$$
\left\{\begin{array}{l}
\left\langle\nabla\left(f \circ \varphi^{-1}\right)(\bar{z}), d\right\rangle<0 \\
\left\langle\nabla\left(g_{t} \circ \varphi^{-1}\right)(\bar{z}), d\right\rangle \leq 0, \quad \forall t \in T_{\varphi^{-1}}(\bar{z}) .
\end{array}\right.
$$


Moreover, we deduce from Lemma 2.7 that $\operatorname{co} \nabla\left(f \circ \varphi^{-1}\right)(\bar{z})$ is a compact set, and hence, $\operatorname{co} \nabla\left(f \circ \varphi^{-1}\right)(\bar{z})+\Delta_{\varphi^{-1}}$ is closed. According to Lemma 2.6, one gets

$$
0 \in \operatorname{co} \nabla\left(f \circ \varphi^{-1}\right)(\bar{z})+\operatorname{pos} \bigcup_{t \in T_{E}(\bar{z})} \nabla\left(g_{t} \circ E\right)(\bar{z}) .
$$

In view of Lemma 2.5 , there exists $\lambda \in \Lambda_{\varphi^{-1}}(\bar{z})$ such that

$$
\nabla\left(f \circ \varphi^{-1}\right)(\bar{z})+\sum_{t \in T} \lambda_{t} \nabla\left(g_{t} \circ \varphi^{-1}\right)(\bar{z})=0 .
$$

This completes the proof.

Corollary 3.7. (i) If $(U, \varphi)$ be an arbitrary chart in $\mathscr{A}, \bar{x} \in \Omega_{\varphi}$ is a locally optimal solution of $\left(P_{\varphi}\right), \bar{z}=\varphi(\bar{x})$ and $\left(A C Q_{\varphi^{-1}}\right)$ holds at $\bar{z}$, then there exists $\lambda \in \Lambda_{\varphi^{-1}}(\bar{z})$ such that

$$
\nabla\left(f \circ \varphi^{-1}\right)(\bar{z})+\sum_{t \in T} \lambda_{t} \nabla\left(g_{t} \circ \varphi^{-1}\right)(\bar{z})=0 .
$$

(ii) If $\bar{x} \in \Omega$ is a locally optimal solution of $(P),(U, \varphi)$ is an arbitrary chart about $\bar{x}, \bar{z}=\varphi(\bar{x})$ and $\left(A C Q_{\varphi^{-1}}\right)$ holds at $\bar{z}$, then there exists $\lambda \in \Lambda_{\varphi^{-1}}(\bar{z})$ such that

$$
\nabla\left(f \circ \varphi^{-1}\right)(\bar{z})+\sum_{t \in T} \lambda_{t} \nabla\left(g_{t} \circ \varphi^{-1}\right)(\bar{z})=0 .
$$

Example 3.8. Let $\mathscr{E}=\mathbb{R}^{2}$ and $M^{1}=\mathbb{S}^{1}=\left\{x \in \mathbb{R}^{2} \mid x_{1}^{2}+x_{2}^{2}=1\right\}$. Then, $M^{1}$ is a smooth manifold with the atlas $\mathscr{A}=\left\{\left(U_{i}, \varphi_{i}\right), i=1,2\right\}$, where

$$
\begin{gathered}
U_{1}=\mathbb{S}^{1} \backslash\{(0,1)\}, \varphi_{1}\left(x_{1}, x_{2}\right)=\frac{x_{1}}{1-x_{2}}, \varphi_{1}^{-1}(z)=\left(\frac{2 z}{z^{2}+1}, \frac{z^{2}-1}{z^{2}+1}\right) \\
U_{2}=\mathbb{S}^{1} \backslash\{(0,-1)\}, \varphi_{2}\left(x_{1}, x_{2}\right)=\frac{x_{1}}{1+x_{2}}, \varphi_{2}^{-1}(z)=\left(\frac{2 z}{z^{2}+1},-\frac{z^{2}-1}{z^{2}+1}\right) .
\end{gathered}
$$

Consider the following problem

$$
\begin{aligned}
& \text { (P) }: \min f(x)=x_{2}^{2} \\
& \text { s.t. } \quad g_{t}(x)=\left(x_{2}-\frac{4}{5}\right)\left(x_{2}-t\right) \leq 0, t \in T=\left[0, \frac{1}{2}\right], \\
& \quad x \in \mathbb{S}^{1}=\left\{x \in \mathbb{R}^{2} \mid x_{1}^{2}+x_{2}^{2}=1\right\} .
\end{aligned}
$$

Then, $\Omega=\left\{x \in \mathbb{S}^{1} \mid \frac{1}{2} \leq x_{2} \leq \frac{4}{5}\right\}$ and $\bar{x}=\left( \pm \frac{\sqrt{3}}{2}, \frac{1}{2}\right)$ are optimal solutions of $(P)$. For $\hat{x}=\left(\frac{3}{5}, \frac{4}{5}\right) \in$ $\Omega$, one has $\hat{x} \in U_{1}$. Consider the semi-infinite programmings with respect to $\varphi_{1}$ as follows.

$$
\begin{gathered}
\left(P_{\varphi_{1}}\right): \min _{x \in U_{1}}\left\{f(x)=x_{2}^{2} \mid g_{t}(x)=\left(x_{2}-\frac{4}{5}\right)\left(x_{2}-t\right) \leq 0, t \in T\right\}, \Omega_{\varphi_{1}}=\left\{x \in U_{1} \mid \frac{1}{2} \leq x_{2} \leq \frac{4}{5}\right\}, \\
\left(P_{\varphi_{1}^{-1}}\right): \min _{z \in \varphi_{1}\left(U_{1}\right)}\left\{\left(f \circ \varphi_{1}^{-1}\right)(z)=\left(\frac{z^{2}-1}{z^{2}+1}\right)^{2} \mid\left(g_{t} \circ \varphi_{1}^{-1}\right)(z)=\left(\frac{z^{2}-1}{z^{2}+1}-\frac{4}{5}\right)\left(\frac{z^{2}-1}{z^{2}+1}-t\right) \leq 0, t \in T\right\} .
\end{gathered}
$$

Then, $\Omega_{\varphi_{1}^{-1}}=\left\{z \in \mathbb{R} \mid \frac{1}{2} \leq \frac{z^{2}-1}{z^{2}+1} \leq \frac{4}{5}\right\}=(-\infty,-\sqrt{3}] \cup[\sqrt{3}, \infty)$ and $\hat{z}=\varphi_{1}(\hat{x})=3$. By some calculations, one has

$$
\begin{gathered}
T_{\varphi_{1}^{-1}}(\hat{z})=\left[0, \frac{1}{2}\right], \nabla\left(f \circ \varphi_{1}^{-1}\right)(z)=\frac{8 z\left(z^{2}-1\right)}{\left(z^{2}+1\right)^{3}}, \nabla\left(f \circ \varphi_{1}^{-1}\right)(\hat{z})=\frac{24}{125}, \\
\nabla\left(g \circ \varphi_{1}^{-1}\right)(z)=\frac{8 z\left(z^{2}-1\right)}{\left(z^{2}+1\right)^{3}}-\left(\frac{4}{5}+t\right) \frac{4 z}{\left(z^{2}+1\right)^{2}}, \nabla\left(g_{t} \circ \varphi_{1}^{-1}\right)(\hat{z})=-\frac{3}{25} t+\frac{12}{125},
\end{gathered}
$$




$$
\begin{gathered}
\left(\bigcup_{t \in\left[0, \frac{1}{2}\right]} \nabla\left(g_{t} \circ \varphi_{1}^{-1}\right)(\hat{z})\right)^{-}=\left(\left[\frac{9}{250}, \frac{12}{125}\right]\right)^{-}=-\mathbb{R}_{+}, \\
\mathscr{T}\left(\Omega_{\varphi_{1}^{-1}}, \hat{z}\right)=\mathbb{R}, \operatorname{pos} \bigcup_{t \in T_{\varphi_{1}^{-1}}(\hat{z})} \nabla\left(g_{t} \circ \varphi_{1}^{-1}\right)(\hat{z})=\mathbb{R}_{+}
\end{gathered}
$$

is closed, i.e., $\left(A C Q_{\varphi^{-1}}\right)$ holds at $\hat{z}$. Moreover,

$$
\nabla f(\hat{z})+\sum_{t \in T} \lambda_{t} \nabla\left(g_{t} \circ \varphi^{-1}\right)(\hat{z})=\frac{24}{125}+\sum_{t \in T} \lambda_{t}\left(-\frac{3}{25} t+\frac{12}{125}\right)>0, \forall \lambda \in \Lambda_{\varphi^{-1}}(\hat{z}) .
$$

Applying Corollary 3.7, we conclude that $\hat{x}$ is not a locally optimal solution of $(P)$.

Proposition 3.9. Let $(U, \varphi)$ be an arbitrary chart and $\bar{z} \in \Omega_{\varphi^{-1}}$. Assume that there exists $\lambda \in \Lambda_{\varphi^{-1}}(\bar{z})$ such that

$$
\nabla\left(f \circ \varphi^{-1}\right)(\bar{z})+\sum_{t \in T} \lambda_{t} \nabla\left(g_{t} \circ \varphi^{-1}\right)(\bar{z})=0 .
$$

If $f, g_{t}(t \in T)$ are $\varphi^{-1}$-convex at $\bar{z}$ on $\Omega_{\varphi^{-1}}$, then $\bar{z}$ is an optimal solution of $\left(P_{\varphi^{-1}}\right)$ and $\varphi^{-1}(\bar{z})$ is an optimal solution of $\left(P_{\varphi}\right)$.

Proof. Since $\bar{z} \in \Omega_{\varphi^{-1}}$ satisfying (3.2), there exists a finite subset $J_{\varphi^{-1}}$ of $T_{\varphi^{-1}}(\bar{z})$ such that

$$
\nabla\left(f \circ \varphi^{-1}\right)(\bar{z})=-\sum_{t \in J_{\varphi^{-1}}} \lambda_{t} \nabla\left(g_{t} \circ \varphi^{-1}\right)(\bar{z}) .
$$

Picking an arbitrary $z \in \Omega_{\varphi^{-1}}$, we get $\left(g_{t} \circ \varphi^{-1}\right)(z) \leq 0=\left(g_{t} \circ \varphi^{-1}\right)(\bar{z}), \forall t \in J_{\varphi^{-1}}$. Thus, by the $\varphi^{-1}$-convexity of $g_{t}(t \in T)$ at $\bar{z}$, we have

$$
\sum_{t \in J_{\varphi^{-1}}} \lambda_{t}\left\langle\nabla\left(g_{t} \circ \varphi^{-1}\right)(\bar{z}), z-\bar{z}\right\rangle \leq \sum_{t \in J_{\varphi^{-1}}} \lambda_{t}\left(\left(g_{t} \circ \varphi^{-1}\right)(z)-\left(g_{t} \circ \varphi^{-1}\right)(\bar{z})\right) \leq 0 .
$$

It follows from the above inequality and (3.2) that

$$
\left\langle\nabla\left(f \circ \varphi^{-1}\right)(\bar{z}), z-\bar{z}\right\rangle=-\sum_{t \in J_{\varphi^{-1}}} \lambda_{t}\left\langle\nabla\left(g_{t} \circ \varphi^{-1}\right)(\bar{z}), z-\bar{z}\right\rangle \geq 0,
$$

which together with the $\varphi^{-1}$-convexity of $f$ at $\bar{z}$ implies

$$
\left(f \circ \varphi^{-1}\right)(z) \geq\left(f \circ \varphi^{-1}\right)(\bar{z}), \forall z \in \Omega_{\varphi^{-1}} .
$$

The proof is complete.

Example 3.10. Let $\mathscr{E}=\mathbb{R}^{2}$ and $M^{2}=\operatorname{int} \mathbb{R}_{+}^{2}=\left\{x=\left(x_{1}, x_{2}\right) \in \mathbb{R}^{2} \mid x_{1}>0, x_{2}>0\right\}$. Then, $M^{2}$ is a smooth manifold with single chart $(U, \varphi)$, where

$$
U=M^{2}, \varphi(x)=\left(\sqrt[3]{x_{1}-2}, x_{2}\right), \varphi^{-1}(z)=\left(z_{1}^{3}+2, z_{2}\right) .
$$

Consider the following nonconvex and nonsmooth semi-infinite programming

$$
\begin{aligned}
& (P): \min f(x)=\sqrt[3]{x_{1}-2}+\frac{1}{4} x_{2}^{4} \\
& \text { s.t. } \quad g_{t}(x)=-t \sqrt[3]{x_{1}-2}+(t-1)\left(x_{2}-1\right) \leq 0, \quad t \in T=[0,1],
\end{aligned}
$$$$
x \in M^{2}=\left\{x \in \mathbb{R}^{2} \mid x_{1}>0, x_{2}>0\right\} .
$$

We can check that $\Omega=\left\{x \in \mathbb{R}^{2} \mid x_{1} \geq 2, x_{2} \geq 1\right\}$. Hence, $\left(P_{\varphi}\right)$ coincides with $(P)$ and 


$$
\left(P_{\varphi^{-1}}\right): \min \left(f \circ \varphi^{-1}\right)(z)=z_{1}+\frac{1}{4} z_{2}^{4}
$$

s.t. $\quad\left(g_{t} \circ \varphi^{-1}\right)(z)=-t z_{1}+(t-1)\left(z_{2}-1\right) \leq 0, \quad t \in T=[0,1]$.

Therefore, $\Omega_{\varphi^{-1}}=\left\{z \in \mathbb{R}^{2} \mid z_{1} \geq 0, z_{2} \geq 1\right\}$. Let us take $\bar{z}=(0,1) \in \Omega_{\varphi^{-1}}$. We can check that $\bar{z}$ is an optimal solution of $\left(P_{\varphi^{-1}}\right)$. Moreover, by some calculations, one has

$$
\begin{gathered}
\nabla\left(f \circ \varphi^{-1}\right)(\bar{z})=(1,1), T_{\varphi^{-1}}(\bar{z})=T, \\
\nabla\left(g_{t} \circ \varphi^{-1}\right)(z)=(-t, t-1), \forall t \in T, \bigcup_{t \in T_{\varphi^{-1}}(\bar{z})} \nabla\left(g_{t} \circ \varphi^{-1}\right)(\bar{z})=[-1,0] \times[-1,0] \\
\operatorname{pos} \bigcup_{t \in T_{\varphi^{-1}}(\bar{z})} \nabla\left(g_{t} \circ \varphi^{-1}\right)(\bar{z})=-\mathbb{R}_{+}^{2},\left(\bigcup_{t \in T_{\varphi^{-1}}(\bar{z})} \nabla\left(g_{t} \circ \varphi^{-1}\right)(\bar{z})\right)^{-}=\mathbb{R}_{+}^{2}, \mathscr{T}\left(\Omega_{\varphi^{-1}}, \bar{z}\right)=\mathbb{R}_{+}^{2} .
\end{gathered}
$$

Thus, $\left(A C Q_{\varphi^{-1}}\right)$ holds at $\bar{z}$ and all assumptions in Proposition 3.6 are satisfied. Now, let $\lambda: T \rightarrow$ $\mathbb{R}$ be defined by $\lambda(t)$ equal to 2 if $t=1 / 2$; and equal to zero otherwise. Then, $\lambda \in \Lambda_{\varphi^{-1}}(\bar{z})$ and

$$
\nabla\left(f \circ \varphi^{-1}\right)(\bar{z})+\sum_{t \in T} \lambda_{t} \nabla\left(g_{t} \circ \varphi^{-1}\right)(\bar{z})=(1,1)+2 .\left(-\frac{1}{2},-\frac{1}{2}\right)=(0,0),
$$

i.e., the conclusion of Proposition 3.6 is satisfied.

Moreover, we can check that $f, g_{t}(t \in T)$ are $\varphi^{-1}$-convex at $\bar{z}$ on $\Omega_{\varphi^{-1}}$. Hence, all assumptions in Proposition 3.9 hold. Then, it follows that $\bar{z}$ is an optimal solution of $\left(P_{\varphi^{-1}}\right)$. By virtue of Lemma 3.3, one concludes that $\bar{x}=\varphi^{-1}(\bar{z})=(2,1)$ is an optimal solution of $(P)$.

\section{Duality}

4.1. Lagrange duality. Let $(U, \varphi)$ be an arbitrary chart. For $z \in \varphi(U)$ and $\lambda \in \mathbb{R}_{+}^{|T|}$, denote

$$
\mathscr{L}_{\varphi^{-1}}(z, \lambda):=\left(f \circ \varphi^{-1}\right)(z)+\sum_{t \in T} \lambda_{t}\left(g_{t} \circ \varphi^{-1}\right)(z),
$$

and $\phi(\lambda)=\min _{z \in \varphi(U)} \mathscr{L}_{\varphi^{-1}}(z, \lambda)$.

We present the Lagrange (see, e.g., [36]) type dual problem as follows:

$\left(D_{L_{\varphi^{-1}}}\right): \max \phi(\lambda), \lambda \in \mathbb{R}_{+}^{|T|}$.

Proposition 4.1. (Weak duality between $\left(P_{\varphi^{-1}}\right)$ and $\left(D_{L_{\varphi^{-1}}}\right)$ ) If $z \in \Omega_{\varphi^{-1}}$ and $\lambda \in \mathbb{R}_{+}^{|T|}$, then

$$
\left(f \circ \varphi^{-1}\right)(z) \geq \phi(\lambda) .
$$

Proof. Since $z \in \Omega_{\varphi^{-1}}$ and $\lambda \in \mathbb{R}_{+}^{|T|}$, one has

$$
\begin{gathered}
\left(g_{t} \circ \varphi^{-1}\right)(z) \leq 0, \forall t \in T, \\
\phi(\lambda)=\min _{z \in \varphi(U)} \mathscr{L}_{\varphi^{-1}}(z, \lambda) \leq\left(f \circ \varphi^{-1}\right)(z)+\sum_{t \in T} \lambda_{t}\left(g_{t} \circ \varphi^{-1}\right)(z) .
\end{gathered}
$$

We deduce from (4.1) that $\sum_{t \in T} \lambda_{t}\left(g_{t} \circ \varphi^{-1}\right)(z) \leq 0$. Hence,

$$
\phi(\lambda) \leq\left(f \circ \varphi^{-1}\right)(z)+\sum_{t \in T} \lambda_{t}\left(g_{t} \circ \varphi^{-1}\right)(z) \leq\left(f \circ \varphi^{-1}\right)(z),
$$

which guarantees the conclusion. 
Corollary 4.2. (Weak duality between $\left(P_{\varphi}\right)$ and $\left(D_{L_{\varphi^{-1}}}\right)$ ) If $x \in \Omega_{\varphi}$ and $\lambda \in \mathbb{R}_{+}^{|T|}$, then $f(x) \geq$ $\phi(\lambda)$.

Proposition 4.3. (Strong duality between $\left(P_{\varphi^{-1}}\right)$ and $\left(D_{L_{\varphi^{-1}}}\right)$ ) Let $\bar{z}$ be an optimal solution of $\left(P_{\varphi^{-1}}\right)$, and let $\left(A C Q_{\varphi^{-1}}\right)$ hold at $\bar{z}$. If $f, g_{t}(t \in T)$ are $\varphi^{-1}$-convex at $\bar{z}$, then there exists $\bar{\lambda} \in \mathbb{R}_{+}^{|T|}$ such that $\bar{\lambda}$ is an optimal solution of $\left(D_{L_{\varphi^{-1}}}\right)$, and $\left(f \circ \varphi^{-1}\right)(\bar{z})=\phi(\bar{\lambda})$.

Proof. By Proposition 3.6, there exists $\bar{\lambda} \in \Lambda_{\varphi^{-1}}(\bar{z})$ such that

$$
\nabla\left(f \circ \varphi^{-1}\right)(\bar{z})+\sum_{t \in T} \bar{\lambda}_{t} \nabla\left(g_{t} \circ \varphi^{-1}\right)(\bar{z})=0 .
$$

As $\bar{\lambda} \in \Lambda_{\varphi^{-1}}(\bar{z}), \bar{\lambda}_{t}\left(g_{t} \circ \varphi^{-1}\right)(\bar{z})=0$ for all $t \in T$. Thus,

$$
\left(f \circ \varphi^{-1}\right)(\bar{z})=\left(f \circ \varphi^{-1}\right)(\bar{z})+\sum_{t \in T} \bar{\lambda}_{t}\left(g_{t} \circ \varphi^{-1}\right)(\bar{z})=\mathscr{L}_{\varphi^{-1}}(\bar{z}, \bar{\lambda}) .
$$

Since $f, g_{t}(t \in T)$ are $\varphi^{-1}$-convex and $\bar{z}$ on $\Omega_{\varphi^{-1}}$, we get that, for all $z \in \Omega_{\varphi^{-1}}$,

$$
\left(f \circ \varphi^{-1}\right)(z)-\left(f \circ \varphi^{-1}\right)(\bar{z}) \geq\left\langle\nabla\left(f \circ \varphi^{-1}\right)(\bar{z}), z-\bar{z}\right\rangle,
$$

and

$$
\left(g_{t} \circ \varphi^{-1}\right)(z)-\left(g_{t} \circ \varphi^{-1}\right)(\bar{z}) \geq\left\langle\nabla\left(g_{t} \circ \varphi^{-1}\right)(\bar{z}), z-\bar{z}\right\rangle, t \in T .
$$

It follows from the above inequalities and (4.2) that

$$
\begin{gathered}
\left(\left(f \circ \varphi^{-1}\right)(z)+\sum_{t \in T} \bar{\lambda}_{t}\left(g_{t} \circ \varphi^{-1}\right)(z)\right)-\left(\left(f \circ \varphi^{-1}\right)(\bar{z})+\sum_{t \in T} \bar{\lambda}_{t}\left(g_{t} \circ \varphi^{-1}\right)(\bar{z})\right) \\
\geq\left\langle\nabla\left(f \circ \varphi^{-1}\right)(\bar{z})+\sum_{t \in T} \bar{\lambda}_{t} \nabla\left(g_{t} \circ \varphi^{-1}\right)(\bar{z}), z-\bar{z}\right\rangle=0,
\end{gathered}
$$

or equivalently,

$$
\mathscr{L}_{\varphi^{-1}}(z, \bar{\lambda}) \geq \mathscr{L}_{\varphi^{-1}}(\bar{z}, \bar{\lambda})
$$

This, along with (4.3), tells us that

$$
\left(f \circ \varphi^{-1}\right)(\bar{z})=\mathscr{L}_{\varphi^{-1}}(\bar{z}, \bar{\lambda})=\min _{z \in \varphi(U)} \mathscr{L}_{\varphi^{-1}}(z, \bar{\lambda})=\phi(\bar{\lambda}) .
$$

Moreover, by invoking Proposition 4.1, we derive from the above inequality that

$$
\phi(\lambda) \leq\left(f \circ \varphi^{-1}\right)(\bar{z})=\phi(\bar{\lambda}), \forall \lambda \in \mathbb{R}_{+}^{|T|} .
$$

So, there exists $\bar{\lambda} \in \mathbb{R}_{+}^{|T|}$ is an optimal solution of $\left(D_{L_{\varphi^{-1}}}\right)$ and $\left(f \circ \varphi^{-1}\right)(\bar{z})=\phi(\bar{\lambda})$.

Corollary 4.4. (i) (Strong duality between $\left(P_{\varphi}\right)$ and $\left(D_{L_{\varphi^{-1}}}\right)$ ) Suppose that $\bar{x}$ be an optimal solution of $\left(P_{\varphi}\right)$, and $z=\varphi(\bar{x}),\left(A C Q_{\varphi^{-1}}\right)$ holds at $\bar{z}$. If $f, g_{t}(t \in T)$ are $\varphi^{-1}$-convex at $\bar{z}$, then there exists $\bar{\lambda} \in \mathbb{R}_{+}^{|T|}$ such that $\bar{\lambda}$ is an optimal solution of $\left(D_{L_{\varphi^{-1}}}\right)$ and $f(\bar{x})=\phi(\bar{\lambda})$.

(ii) (Strong duality between $(P)$ and $\left.\left(D_{L_{\varphi^{-1}}}\right)\right)$ Suppose that $\bar{x}$ be an optimal solution of $(P),(U, \varphi)$ is an arbitrary chart about $\bar{x}, z=\varphi(\bar{x}),\left(A C Q_{\varphi^{-1}}\right)$ holds at $\bar{z}$. If $f, g_{t}(t \in T)$ are $\varphi^{-1}$-convex at $\bar{z}$, then there exists $\bar{\lambda} \in \mathbb{R}_{+}^{|T|}$ such that $\bar{\lambda}$ is an optimal solution of $\left(D_{L_{\varphi^{-1}}}\right)$ and $f(\bar{x})=\phi(\bar{\lambda})$. 
Example 4.5. Let $\mathscr{E}=\mathbb{R}^{2}$ and $M^{2}=\operatorname{int} \mathbb{R}_{+}^{2}=\left\{x=\left(x_{1}, x_{2}\right) \in \mathbb{R}^{2} \mid x_{1}>0, x_{2}>0\right\}$. Then, $M^{2}$ is a smooth manifold with the single chart $(U, \varphi)$, where

$$
U=M^{2}, \varphi(x)=\left(\sqrt[3]{x_{1}-2}, x_{2}\right), \varphi^{-1}(z)=\left(z_{1}^{3}+2, z_{2}\right) .
$$

Consider the following nonconvex and nonsmooth semi-infinite programming

$(P): \min f(x)=\sqrt[3]{\left(x_{1}-2\right)^{2}}+\frac{1}{2}\left(x_{2}-1\right)^{2}$

s.t. $\quad g_{t}(x)=-\sqrt[3]{x_{1}-2}+t \leq 0, \quad t \in T=[0,1]$,

$x \in M^{2}=\left\{x \in \mathbb{R}^{2} \mid x_{1}>0, x_{2}>0\right\}$.

We can verify that $\Omega=\left\{x \in \mathbb{R}^{2} \mid x_{1} \geq 3, x_{2}>0\right\}$. Then, $\left(P_{\varphi}\right)$ coincides with $(P)$ and

$\left(P_{\varphi^{-1}}\right): \min \left(f \circ \varphi^{-1}\right)(z)=z_{1}^{2}+\frac{1}{2}\left(z_{2}-1\right)^{2}$

s.t. $\quad\left(g_{t} \circ \varphi^{-1}\right)(z)=-z_{1}+t \leq 0, \quad t \in T=[0,1]$.

Therefore, $\Omega_{\varphi^{-1}}=\left\{z \in \mathbb{R}^{2} \mid z_{1} \geq 1, z_{2}>0\right\}$. For $z \in \varphi(U)$ and $\lambda \in \mathbb{R}_{+}^{|T|}$,

$\mathscr{L}_{\varphi^{-1}}(z, \lambda)=z_{1}^{2}+\frac{1}{2}\left(z_{2}-1\right)^{2}+\sum_{t \in T} \lambda_{t}\left(-z_{1}+t\right)=\left(z_{1}-\frac{1}{2} \sum_{t \in T} \lambda_{t}\right)^{2}+\frac{1}{2}\left(z_{2}-1\right)^{2}+\sum_{t \in T} \lambda_{t} t-\frac{1}{4} \sum_{t \in T} \lambda_{t}^{2}$.

Hence,

$\left(D_{L_{\varphi^{-1}}}\right): \max \phi(\lambda)=\sum_{t \in T} \lambda_{t} t-\frac{1}{4} \sum_{t \in T} \lambda_{t}^{2}, \lambda \in \mathbb{R}_{+}^{|T|}$.

Let us take $\bar{z}=(1,1) \in \Omega_{\varphi^{-1}}$. We can check that $\bar{z}$ is an optimal solution of $\left(P_{\varphi^{-1}}\right)$. Moreover, by some calculations, one has

$$
\begin{gathered}
\nabla\left(f \circ \varphi^{-1}\right)(\bar{z})=(2,0), T_{\varphi^{-1}}(\bar{z})=\{1\}, \\
\nabla\left(g_{t} \circ \varphi^{-1}\right)(\bar{z})=(-1,0), \forall t \in T,\left(\bigcup_{t \in T_{\varphi^{-1}}(\bar{z})} \nabla\left(g_{t} \circ \varphi^{-1}\right)(\bar{z})\right)^{-}=\mathbb{R}_{+} \times \mathbb{R}, \\
\mathscr{T}\left(\Omega_{\varphi^{-1}}, \bar{z}\right)=\mathbb{R}_{+} \times \mathbb{R}, \operatorname{pos} \bigcup_{t \in T_{\varphi^{-1}}(\bar{z})} \nabla\left(g_{t} \circ \varphi^{-1}\right)(\bar{z})=-\mathbb{R}_{+} \times\{0\} .
\end{gathered}
$$

Thus, $\left(A C Q_{\varphi^{-1}}\right)$ holds at $\bar{z}$ and all suppositions in Proposition 3.6 are fulfilled. Now, let $\bar{\lambda}: T \rightarrow$ $\mathbb{R}$ be defined by $\bar{\lambda}(t)$ equal to 2 if $t=1$; and equal to zero otherwise. Then, $\bar{\lambda} \in \Lambda_{\varphi^{-1}}(\bar{z})$ and

$$
\nabla\left(f \circ \varphi^{-1}\right)(\bar{z})+\sum_{t \in T} \bar{\lambda}_{t} \nabla\left(g_{t} \circ \varphi^{-1}\right)(\bar{z})=(2,0)+2 .(-1,0)=(0,0) .
$$

Moreover, we can check that $f, g_{t}(t \in T)$ are $\varphi^{-1}$-convex at $\bar{z}$ on $\Omega_{\varphi^{-1}}$. Hence, all hypothesis in Proposition 4.3 hold. Then, it follows that $\bar{\lambda}$ is an optimal solution of $\left(D_{L_{\varphi^{-1}}}\right)$ and $\left(f \circ \varphi^{-1}\right)(\bar{z})=$ $\phi(\bar{\lambda})=1$. We can check directly that $\phi(\bar{\lambda})=2 \cdot 1-\frac{1}{4} \cdot 2^{2}=1$.

4.2. Mond-Weir duality. Let $(U, \varphi)$ be an arbitrary chart. For $u \in \varphi(U)$ and $\lambda \in \mathbb{R}_{+}^{|T|}$, denote

$$
\widehat{\mathscr{L}}_{\varphi^{-1}}(u, \lambda):=\left(f \circ \varphi^{-1}\right)(u) .
$$

We consider the $\varphi^{-1}$-Mond-Weir [37] dual problem $\left(D_{M W_{\varphi^{-1}}}\right)$ of $\left(P_{\varphi}\right)$ as follows

$$
\begin{aligned}
& \max \widehat{\mathscr{L}}_{\varphi^{-1}}(u, \lambda)=\left(f \circ \varphi^{-1}\right)(u) \\
& \text { s.t. } \quad \nabla\left(f \circ \varphi^{-1}\right)(u)+\sum_{t \in T} \lambda_{t} \nabla\left(g_{t} \circ \varphi^{-1}\right)(u)=0, \\
& \sum_{t \in T} \lambda_{t}\left(g_{t} \circ \varphi^{-1}\right)(u) \geq 0, u \in \varphi(U), \lambda \in \mathbb{R}_{+}^{|T|} .
\end{aligned}
$$


The feasible set of $\left(D_{M W_{\varphi^{-1}}}\right)$ is

$$
\begin{aligned}
\Omega_{M W_{\varphi^{-1}}}:= & \left\{(u, \lambda) \in \varphi(U) \times \mathbb{R}_{+}^{|T|} \mid \nabla\left(f \circ \varphi^{-1}\right)(u)+\sum_{t \in T} \lambda_{t} \nabla\left(g_{t} \circ \varphi^{-1}\right)(u)=0,\right. \\
& \left.\sum_{t \in T} \lambda_{t}\left(g_{t} \circ \varphi^{-1}\right)(u) \geq 0\right\} .
\end{aligned}
$$

and its projection on $\mathbb{R}^{n}$ is $Y_{M W_{\varphi^{-1}}}:=\left\{u \in \varphi(U) \mid(u, \lambda) \in \Omega_{M W_{\varphi^{-1}}}\right\}$.

Definition 4.6. The point $(\bar{u}, \bar{\lambda}) \in \Omega_{M W_{\varphi^{-1}}}$ is said to be an optimal solution of $\left(D_{M W_{\varphi^{-1}}}\right)$ if

$$
\widehat{\mathscr{L}}_{\varphi^{-1}}(\bar{u}, \bar{\lambda}) \geq \widehat{\mathscr{L}}_{\varphi^{-1}}(u, \lambda), \forall(\bar{u}, \bar{\lambda}) \in \Omega_{M W_{\varphi^{-1}}} .
$$

Proposition 4.7. (Weak duality between $\left(P_{\varphi^{-1}}\right)$ and $\left(D_{M W_{\varphi^{-1}}}\right)$ ) Let $z \in \Omega_{\varphi^{-1}}$ and $(u, \lambda) \in$ $\Omega_{M W_{\varphi^{-1}}}$. If $f, g_{t}(t \in T)$ are $\varphi^{-1}$-convex at $u$ on $\Omega_{M W_{\varphi}^{-1}} \cup Y_{M W_{\varphi}^{-1}}$, then $\left(f \circ \varphi^{-1}\right)(z) \geq \widehat{\mathscr{L}}_{\varphi^{-1}}(u, \lambda)$. Proof. Since $z \in \Omega_{\varphi^{-1}}$ and $(u, \lambda) \in \Omega_{M W_{M W_{\varphi^{-1}}}}$, we have

$$
\begin{gathered}
\left(g_{t} \circ \varphi^{-1}\right)(z) \leq 0, \forall t \in T, \\
\sum_{t \in T} \lambda_{t}\left(g_{t} \circ \varphi^{-1}\right)(u) \geq 0, \\
\nabla\left(f \circ \varphi^{-1}\right)(u)+\sum_{t \in T} \lambda_{t} \nabla\left(g_{t} \circ \varphi^{-1}\right)(u)=0 .
\end{gathered}
$$

From (4.4) and (4.5), one has $\sum_{t \in T} \lambda_{t}\left(g_{t} \circ \varphi^{-1}\right)(z) \leq 0 \leq \sum_{t \in T} \lambda_{t}\left(g_{t} \circ \varphi^{-1}\right)(u)$. This along with the $\varphi^{-1}$-convexity of $g_{t}(t \in T)$ at $u$ leads that

$$
\sum_{t \in T} \lambda_{t}\left\langle\nabla\left(g_{t} \circ \varphi^{-1}\right)(u), z-u\right\rangle \leq 0
$$

Moreover, since $f$ is $\varphi^{-1}$-convex, we have

$$
\left\langle\nabla\left(f \circ \varphi^{-1}\right)(u), z-u\right\rangle \leq\left(f \circ \varphi^{-1}\right)(z)-\left(f \circ \varphi^{-1}\right)(u)=\left(f \circ \varphi^{-1}\right)(z)-\widehat{\mathscr{L}}_{\varphi^{-1}}(u, \lambda) .
$$

Combing (4.6), (4.7) and (4.8), we have

$$
\left.0=\left\langle\nabla f \circ \varphi^{-1}\right)(u)+\sum_{t \in T} \lambda_{t} \nabla\left(g_{t} \circ \varphi^{-1}\right)(u), z-u\right\rangle \leq\left(f \circ \varphi^{-1}\right)(z)-\widehat{\mathscr{L}}_{\varphi^{-1}}(u, \lambda),
$$

which arrives at the conclusion.

Corollary 4.8. (Weak duality between $\left(P_{\varphi}\right)$ and $\left(D_{M W_{\varphi^{-1}}}\right)$ ) Let $x \in \Omega_{\varphi}$ and $(u, \lambda) \in \Omega_{M W_{\varphi^{-1}}}$. If $f, g_{t}(t \in T)$ are $\varphi^{-1}$-convex at $u$ on $\Omega_{M W_{\varphi^{-1}}} \cup Y_{M W_{\varphi^{-1}}}$, then $f(x) \geq \widehat{\mathscr{L}}_{\varphi^{-1}}(u, \lambda)$.

Proof. Let $x \in \Omega_{\varphi}$ and $(u, \lambda) \in \Omega_{M W_{\varphi^{-1}}}$. Since $\varphi^{-1}\left(\Omega_{\varphi^{-1}}\right)=\Omega_{\varphi}$, there exists $z \in \Omega_{\varphi^{-1}}$ such that $x=\varphi^{-1}(z)$ and $f(x)=\left(f \circ \varphi^{-1}\right)(z)$. The conclusion follows from Proposition 4.7.

Proposition 4.9. (Strong duality between $\left(P_{\varphi^{-1}}\right)$ and $\left(D_{M W_{\varphi^{-1}}}\right)$ ) Let $\bar{z}$ be an optimal solution of $\left(P_{\varphi^{-1}}\right)$ and $\left(A C Q_{\varphi^{-1}}\right)$ hold at $\bar{z}$. Then, there exists $\bar{\lambda} \in \Lambda_{\varphi^{-1}}(\bar{z})$ such that $(\bar{z}, \bar{\lambda}) \in \Omega_{M W_{\varphi^{-1}}}$ and $\left(f \circ \varphi^{-1}\right)(\bar{z})=\widehat{\mathscr{L}}_{\varphi^{-1}}(\bar{z}, \bar{\lambda})$. Furthermore, if $f, g_{t}(t \in T)$ are $\varphi^{-1}$-convex at $\bar{z}$ on $\Omega_{M W_{\varphi^{-1}}} \cup$ $Y_{M W_{\varphi^{-1}}}$, then $(\bar{z}, \bar{\lambda})$ is an optimal solution of $\left(D_{M W_{\varphi^{-1}}}\right)$. 
Proof. By Proposition 3.6, there exists $\bar{\lambda} \in \Lambda_{\varphi^{-1}}(\bar{z})$ such that

$$
\nabla\left(f \circ \varphi^{-1}\right)(\bar{z})+\sum_{t \in T} \bar{\lambda}_{t} \nabla\left(g_{t} \circ \varphi^{-1}\right)(\bar{z})=0 .
$$

Since $\bar{\lambda} \in \Lambda_{\varphi^{-1}}(\bar{z}), \bar{\lambda}_{t}\left(g_{t} \circ \varphi^{-1}\right)(\bar{z})=0$ for all $t \in T$, and hence, $\sum_{t \in T} \bar{\lambda}_{t}\left(g_{t} \circ \varphi^{-1}\right)(\bar{z})=0$. Thus, $(\bar{z}, \bar{\lambda}) \in \Omega_{M W_{\varphi^{-1}}},\left(f \circ \varphi^{-1}\right)(\bar{z})=\mathscr{L}_{\varphi^{-1}}(\bar{z}, \bar{\lambda})$ and

$$
\widehat{\mathscr{L}}_{\varphi^{-1}}(\bar{z}, \bar{\lambda})=\left(f \circ \varphi^{-1}\right)(\bar{z}) \geq \widehat{\mathscr{L}}_{\varphi^{-1}}(u, \lambda), \forall(u, \lambda) \in \Omega_{M W_{\varphi^{-1}}},
$$

which completes the proof.

Corollary 4.10. (i) (Strong duality between $\left(P_{\varphi}\right)$ and $\left(D_{M W_{\varphi^{-1}}}\right)$ ) Suppose that $\bar{x}$ be an optimal solution of $\left(P_{\varphi}\right), z=\varphi(\bar{x}),\left(A C Q_{\varphi^{-1}}\right)$ holds at $\bar{z}$. Then, there exists $\bar{\lambda} \in \Lambda_{\varphi^{-1}}(\bar{z})$ such that $(\bar{z}, \bar{\lambda}) \in \Omega_{M W_{\varphi^{-1}}}$. Furthermore, if $f, g_{t}(t \in T)$ are $\varphi^{-1}$-convex at $\bar{z}$ on $\Omega_{M W_{\varphi^{-1}}} \cup Y_{M W_{\varphi^{-1}}}$, then $(\bar{z}, \bar{\lambda})$ is an optimal solution of $\left(D_{M W_{\varphi^{-1}}}\right)$.

(ii) (Strong duality between $(P)$ and $\left(D_{M W_{\varphi^{-1}}}\right)$ ) Suppose that $\bar{x}$ be an optimal solution of $(P)$, $(U, \varphi)$ is an arbitrary chart about $\bar{x}, z=\varphi(\bar{x}),\left(A C Q_{\varphi^{-1}}\right)$ holds at $\bar{z}$. Then, there exists $\bar{\lambda} \in$ $\Lambda_{\varphi^{-1}}(\bar{z})$ such that $(\bar{z}, \bar{\lambda}) \in \Omega_{M W_{\varphi^{-1}}}$. Furthermore, if $f, g_{t}(t \in T)$ are $\varphi^{-1}$-convex at $\bar{z}$ on $\Omega_{M W_{\varphi^{-1}}} \cup$ $Y_{M W_{\varphi^{-1}}}$, then $(\bar{z}, \bar{\lambda})$ is an optimal solution of $\left(D_{M W_{\varphi^{-1}}}\right)$.

4.3. Wolfe duality. Let $(U, \varphi)$ be an arbitrary chart. For $u \in \varphi(U)$ and $\lambda \in \mathbb{R}_{+}^{|T|}$, denote $\widetilde{\mathscr{L}}_{\varphi^{-1}}(u, \lambda):=\left(f \circ \varphi^{-1}\right)(u)+\sum_{t \in T} \lambda_{t}\left(g_{t} \circ \varphi^{-1}\right)(u)$.

We define the Wolfe [38] type dual problem as follows:

$$
\begin{gathered}
\left(D_{W_{\varphi^{-1}}}\right): \max \widetilde{\mathscr{L}}_{\varphi^{-1}}(u, \lambda):=\left(f \circ \varphi^{-1}\right)(u)+\sum_{t \in T} \lambda_{t}\left(g_{t} \circ \varphi^{-1}\right)(u) \\
\text { s.t. } \quad \nabla\left(f \circ \varphi^{-1}\right)(u)+\sum_{t \in T} \lambda_{t} \nabla\left(g_{t} \circ \varphi^{-1}\right)(u)=0
\end{gathered}
$$

$$
u \in \varphi(U), \lambda \in \mathbb{R}_{+}^{|T|} \text {. }
$$

The feasible set of $\left(D_{W_{\varphi^{-1}}}\right)$ is

$$
\Omega_{W_{\varphi^{-1}}}:=\left\{(u, \lambda) \in \varphi(U) \times \mathbb{R}_{+}^{|T|} \mid \nabla\left(f \circ \varphi^{-1}\right)(u)+\sum_{t \in T} \lambda_{t} \nabla\left(g_{t} \circ \varphi^{-1}\right)(u)=0\right\}
$$

and its projection on $\varphi(U)$ is $Y_{W_{\varphi^{-1}}}:=\left\{u \in \varphi(U) \mid(u, \lambda) \in \Omega_{W_{\varphi^{-1}}}\right\}$.

Definition 4.11. The point $(\bar{u}, \bar{\lambda}) \in \Omega_{W_{\varphi^{-1}}}$ is said to an optimal solution of $\left(D_{W_{\varphi^{-1}}}\right)$ if

$$
\widetilde{\mathscr{L}}_{\varphi^{-1}}(\bar{u}, \bar{\lambda}) \geq \widetilde{\mathscr{L}}_{\varphi^{-1}}(u, \lambda), \forall(u, \lambda) \in \Omega_{W_{\varphi^{-1}}} .
$$

Proposition 4.12. (Weak duality between $\left(P_{\varphi^{-1}}\right)$ and $\left(D_{W_{\varphi^{-1}}}\right)$ ) Let $z \in \Omega_{\varphi^{-1}}$ and $(u, \lambda) \in \Omega_{W_{\varphi^{-1}}}$. If $f, g_{t}(t \in T)$ are $\varphi^{-1}$-convex at $u$ on $\Omega_{W_{\varphi^{-1}}} \cup Y_{W_{\varphi^{-1}}}$, then $\left(f \circ \varphi^{-1}\right)(z) \geq \widetilde{\mathscr{L}}_{\varphi^{-1}}(u, \lambda)$.

Proof. Since $z \in \Omega_{\varphi^{-1}}$ and $(u, \lambda) \in \Omega_{W_{\varphi^{-1}}}$, one has

$$
\begin{gathered}
\left(g_{t} \circ \varphi^{-1}\right)(z) \leq 0, \forall t \in T, \\
\nabla\left(f \circ \varphi^{-1}\right)(u)+\sum_{t \in T} \lambda_{t} \nabla\left(g_{t} \circ \varphi^{-1}\right)(u)=0 .
\end{gathered}
$$


Since $f, g_{t}(t \in T)$ are $\varphi^{-1}$-convex and $u$, we have

$$
\left(f \circ \varphi^{-1}\right)(z)-\left(f \circ \varphi^{-1}\right)(u) \geq\left\langle\nabla\left(f \circ \varphi^{-1}\right)(u), z-u\right\rangle,
$$

and

$$
\left(g_{t} \circ \varphi^{-1}\right)(z)-\left(g_{t} \circ \varphi^{-1}\right)(u) \geq\left\langle\nabla\left(g_{t} \circ \varphi^{-1}\right)(u), z-u\right\rangle, t \in T .
$$

Taking into account the above inequalities and (4.10), we arrive at

$$
\begin{gathered}
\left(\left(f \circ \varphi^{-1}\right)(z)+\sum_{t \in T} \lambda_{t}\left(g_{t} \circ \varphi^{-1}\right)(z)\right)-\left(\left(f \circ \varphi^{-1}\right)(u)+\sum_{t \in T} \lambda_{t}\left(g_{t} \circ \varphi^{-1}\right)(u)\right) \\
\geq\left\langle\nabla\left(f \circ \varphi^{-1}\right)(u)+\sum_{t \in T} \lambda_{t} \nabla\left(g_{t} \circ \varphi^{-1}\right)(u), z-u\right\rangle=0 .
\end{gathered}
$$

Combining this and (4.9), one obtains

$$
\left(f \circ \varphi^{-1}\right)(z) \geq\left(f \circ \varphi^{-1}\right)(z)+\sum_{t \in T} \lambda_{t}\left(g_{t} \circ \varphi^{-1}\right)(z) \geq\left(f \circ \varphi^{-1}\right)(u)+\sum_{t \in T} \lambda_{t}\left(g_{t} \circ \varphi^{-1}\right)(u),
$$

which finishes the proof.

Corollary 4.13. (Weak duality between $\left(P_{\varphi}\right)$ and $\left(D_{W_{\varphi^{-1}}}\right)$ ) Let $x \in \Omega_{\varphi}$ and $(u, \lambda) \in \Omega_{W_{\varphi^{-1}}}$. If $f, g_{t}(t \in T)$ are $\varphi^{-1}$-convex at $u$ on $\Omega_{W_{\varphi^{-1}}} \cup Y_{W_{\varphi^{-1}}}$, then $f(x) \geq \widetilde{\mathscr{L}}_{\varphi^{-1}}(u, \lambda)$.

Proposition 4.14. (Strong duality between $\left(P_{\varphi^{-1}}\right)$ and $\left.\left(D_{W_{\varphi^{-1}}}\right)\right)$ Let $\bar{z}$ be an optimal solution of $\left(P_{\varphi^{-1}}\right)$ and $\left(A C Q_{\varphi^{-1}}\right)$ hold at $\bar{z}$. Then, there exists $\bar{\lambda} \in \Lambda_{\varphi^{-1}}(\bar{z})$ such that $(\bar{z}, \bar{\lambda}) \in \Omega_{W_{\varphi^{-1}}}$ and $\left(f \circ \varphi^{-1}\right)(\bar{z})=\widetilde{\mathscr{L}}_{\varphi^{-1}}(\bar{z}, \bar{\lambda})$. Furthermore, if $f, g_{t}(t \in T)$ are $\varphi^{-1}$-convex at $\bar{z}$ on $\Omega_{W_{\varphi^{-1}}} \cup Y_{W_{\varphi^{-1}}}$, then $(\bar{z}, \bar{\lambda})$ is an optimal solution of $\left(D_{W_{\varphi^{-1}}}\right)$.

Proof. By Proposition 3.6, there exists $\bar{\lambda} \in \Lambda_{\varphi^{-1}}(\bar{z})$ such that

$$
\nabla\left(f \circ \varphi^{-1}\right)(\bar{z})+\sum_{t \in T} \bar{\lambda}_{t} \nabla\left(g_{t} \circ \varphi^{-1}\right)(\bar{z})=0 .
$$

Since $\bar{\lambda} \in \Lambda_{\varphi^{-1}}(\bar{z}), \bar{\lambda}_{t}\left(g_{t} \circ \varphi^{-1}\right)(\bar{z})=0$ for all $t \in T$. Thus,

$$
\left(f \circ \varphi^{-1}\right)(\bar{z})=\left(f \circ \varphi^{-1}\right)(\bar{z})+\sum_{t \in T} \bar{\lambda}_{t}\left(g_{t} \circ \varphi^{-1}\right)(\bar{z})=\widetilde{\mathscr{L}}_{\varphi^{-1}}(\bar{z}, \bar{\lambda}),
$$

and hence, $(\bar{z}, \bar{\lambda}) \in \Omega_{W_{\varphi^{-1}}}$ and

$$
\widetilde{\mathscr{L}}_{\varphi^{-1}}(\bar{z}, \bar{\lambda})=\left(f \circ \varphi^{-1}\right)(\bar{z}) \geq \widetilde{\mathscr{L}}_{\varphi^{-1}}(u, \lambda), \forall(u, \lambda) \in \Omega_{W_{\varphi^{-1}}},
$$

which completes the proof.

Corollary 4.15. (i) (Strong duality between $\left(P_{\varphi}\right)$ and $\left(D_{W_{\varphi^{-1}}}\right)$ ) Suppose that $\bar{x}$ be an optimal solution of $\left(P_{\varphi}\right), \bar{z}=\varphi(\bar{x}),\left(A C Q_{\varphi^{-1}}\right)$ holds at $\bar{z}$. Then, there exists $\bar{\lambda} \in \Lambda_{\varphi^{-1}}(\bar{z})$ such that $(\bar{z}, \bar{\lambda}) \in \Omega_{W_{\varphi^{-1}}}$. Furthermore, if $f, g_{t}(t \in T)$ are $\varphi^{-1}$-convex at $\bar{z}$ on $\Omega_{W_{\varphi^{-1}}} \cup Y_{W_{\varphi^{-1}}}$, then $(\bar{z}, \bar{\lambda})$ is an optimal solution of $\left(D_{W_{\varphi^{-1}}}\right)$.

(ii) (Strong duality between $(P)$ and $\left(D_{W_{\varphi^{-1}}}\right)$ ) Suppose that $\bar{x}$ be an optimal solution of $(P)$, $(U, \varphi)$ is an arbitrary chart about $\bar{x}, \bar{z}=\varphi(\bar{x}),\left(A C Q_{\varphi^{-1}}\right)$ holds at $\bar{z}$. Then, there exists $\bar{\lambda} \in$ 
$\Lambda_{\varphi^{-1}}(\bar{z})$ such that $(\bar{z}, \bar{\lambda}) \in \Omega_{W_{\varphi^{-1}}}$. Furthermore, if $f, g_{t}(t \in T)$ are $\varphi^{-1}$-convex at $\bar{z}$ on $\Omega_{W_{\varphi^{-1}}} \cup$ $Y_{W_{\varphi^{-1}}}$, then $(\bar{z}, \bar{\lambda})$ is an optimal solution of $\left(D_{W_{\varphi^{-1}}}\right)$.

Example 4.16. Let $\mathscr{E}=\mathbb{R}^{2}$ and $M^{2}=\operatorname{int} \mathbb{R}_{+}^{2}=\left\{x=\left(x_{1}, x_{2}\right) \in \mathbb{R}^{2} \mid x_{1}>0, x_{2}>0\right\}$. Then, $M^{2}$ is a smooth manifold with the single chart $(U, \varphi)$, where

$$
U=M^{2}, \varphi(x)=\left(\sqrt[3]{x_{1}-2}, x_{2}\right), \varphi^{-1}(z)=\left(z_{1}^{3}+2, z_{2}\right) .
$$

Consider the following nonconvex and nonsmooth semi-infinite programming

$$
\begin{aligned}
& (P): \min f(x)=\sqrt[3]{x_{1}-2}+\frac{1}{4} x_{2}^{4} \\
& \text { s.t. } \quad g_{t}(x)=-t \sqrt[3]{x_{1}-2}+(t-1)\left(x_{2}-1\right) \leq 0, \quad t \in T=[0,1],
\end{aligned}
$$$$
x \in M^{2}=\left\{x \in \mathbb{R}^{2} \mid x_{1}>0, x_{2}>0\right\} .
$$

We can check that $\Omega=\left\{x \in \mathbb{R}^{2} \mid x_{1} \geq 2, x_{2} \geq 1\right\}$. Hence, $\left(P_{\varphi}\right)$ coincides with $(P)$ and

$$
\begin{aligned}
& \left.\left(P_{\varphi^{-1}}\right): \min \left(f \circ \varphi^{-1}\right)\right)(z)=z_{1}+\frac{1}{4} z_{2}^{4} \\
& \text { s.t. } \quad\left(g_{t} \circ \varphi^{-1}\right)(z)=-t z_{1}+(t-1)\left(z_{2}-1\right) \leq 0, \quad t \in T=[0,1] .
\end{aligned}
$$

Therefore, $\Omega_{\varphi^{-1}}=\left\{z \in \mathbb{R}^{2} \mid z_{1} \geq 0, z_{2} \geq 1\right\}$.

$$
\begin{gathered}
\left(D_{W_{\varphi^{-1}}}\right): \max \widehat{\mathscr{L}}_{\varphi^{-1}}(u, \lambda):=u_{1}+\frac{1}{4} u_{2}^{4}+\sum_{t \in T} \lambda_{t}\left(-t u_{1}+(t-1)\left(u_{2}-1\right)\right) \\
\text { s.t. } \quad\left(1, u_{2}^{3}\right)+\sum_{t \in T} \lambda_{t}(-t, t-1)=(0,0) \\
u \in \varphi(U), \lambda \in \mathbb{R}_{+}^{|T|} .
\end{gathered}
$$

Let us take $\bar{z}=(0,1) \in \Omega_{\varphi^{-1}}$. We can check that $\bar{z}$ is an optimal solution of $\left(P_{\varphi^{-1}}\right)$. In view of Example 3.10, all assumptions in Proposition 4.14 are satisfied. Now, let $\bar{\lambda}: T \rightarrow \mathbb{R}$ be defined by $\bar{\lambda}(t)$ equal to 2 if $t=1 / 2$; and equal to zero otherwise. Then, $\bar{\lambda} \in \Lambda_{\varphi^{-1}}(\bar{z})$ and

$$
\nabla\left(f \circ \varphi^{-1}\right)(\bar{z})+\sum_{t \in T} \bar{\lambda}_{t} \nabla\left(g_{t} \circ \varphi^{-1}\right)(\bar{z})=(1,1)+2 \cdot\left(-\frac{1}{2},-\frac{1}{2}\right)=(0,0),
$$

i.e., $(\bar{z}, \bar{\lambda}) \in \Omega_{W_{\varphi^{-1}}}$ and $\left(f \circ \varphi^{-1}\right)(\bar{z})=\widetilde{\mathscr{L}}_{\varphi^{-1}}(\bar{z}, \bar{\lambda})$. Moreover, we can check that $f, g_{t}(t \in T)$ are $\varphi^{-1}$-convex at $\bar{z}$ on $\Omega_{W_{\varphi^{-1}}} \cup Y_{W_{\varphi^{-1}}}$. Hence, it follows from Proposition 4.14 that $(\bar{z}, \bar{\lambda})$ is an optimal solution of $\left(D_{W_{\varphi^{-1}}}\right)$.

\section{REFERENCES}

[1] T.D. Chuong, D.S. Kim, Nonsmooth semi-infinite multiobjective optimization problems, J. Optim. Theory Appl. 160 (2014), 748-762.

[2] T.D. Chuong, J.C. Yao, Isolated and proper efficiencies in semi-infinite vector optimization problems, J. Optim. Theory Appl. 162 (2014), 447-462.

[3] N. Kanzi, Lagrange multiplier rules for non-differentiable DC generalized semi-infinite programming problems. J. Glob. Optim. 56 (2013) 417-430.

[4] N. Kanzi, S. Nobakhtian, Optimality conditions for nonsmooth semi-infinite multiobjective programming, Optim. Lett. 8 (2014), 1517-1528.

[5] L.T. Tung, P.T. Hung, On generalized $\tau^{w}$-contingent epiderivatives in parametric vector optimization problems, Appl. Set-Valued Anal. Optim. 2 (2020), 123-137.

[6] L.T. Tung, Karush-Kuhn-Tucker optimality conditions for nonsmooth multiobjective semidefinite and semiinfinite programming, J. Appl. Numer. Optim. 1 (2019), 63-75.

[7] L.T. Tung, Karush-Kuhn-Tucker optimality conditions and duality for convex semi-infinite programming with multiple interval-valued objective functions, J. Appl. Math. Comput. 62 (2020), 67-91. 
[8] L.T. Tung, Karush-Kuhn-Tucker optimality conditions and duality for multiobjective semi-infinite programming via tangential subdifferentials, Numer. Funct. Anal. Optim. 41 (2020), 659-684.

[9] L.T. Tung, Karush-Kuhn-Tucker optimality conditions and duality for semi-infinite programming problems with vanishing constraints, J. Nonlinear Var. Anal. 4 (2020), 319-336

[10] L.T. Tung, Karush-Kuhn-Tucker optimality conditions and duality for multiobjective semi-infinite programming with vanishing constraints, Ann. Oper. Res. doi: 10.1007/s10479-020-03742-1.

[11] M.A. Goberna, N. Kanzi, Optimality conditions in convex multiobjective SIP, Math. Program. 164 (2017), 67-191.

[12] Y. Nishimori, S. Akaho, Learning algorithms utilizing quasigeodesic flows on the Stiefel manifold, Neurocomputing 67 (2005), 106-135.

[13] P. Turaga, A. Veeraraghavan, A. Srivastava, R. Chellappa, Statistical computations on Grassmann and Stiefel manifolds for image and video-based recognition, IEEE Trans. Pattern Anal. Mach. Intell. 33 (2011), 22732286.

[14] S. Treanta, S. Mititelu, Efficiency for variational control problems on Riemann manifolds with geodesic quasiinvex curvilinear integral functionals, Rev. R. Acad. Cienc. Exactas Fis. Nat. Ser. A Mat. 114 (2020), 113.

[15] C. Li, B.S. Mordukhovich, J. Wang, J.C. Yao, Weak sharp minima on Riemannian manifolds, SIAM J. Optim. 21 (2011), 1523-1560.

[16] W.H. Yang, L.H. Zhang, R. Song, Optimality conditions for the nonlinear programming problems on Riemannian manifolds, Pac. J. Optim. 10 (2014), 415-434.

[17] G. Ruiz-Garzón, R. Osuna-Gómez, J. Ruiz-Zapatero, Necessary and sufficient optimality conditions for vector equilibrium problems on Hadamard manifolds, Symmetry 11 (2019), 1037.

[18] R. Bergmann, R. Herzog, Intrinsic formulation of KKT conditions and constraint qualifications on smooth manifolds. SIAM J. Optim. 29 (2019), 2423-2444.

[19] S. Chen, The KKT optimality conditions for optimization problem with interval-valued objective function on Hadamard manifolds, Optimization doi: 10.1080/02331934.2020.1810248.

[20] J. Borwein, A. S. Lewis, Convex Analysis and Nonlinear Optimization: Theory and Examples, Springer, New York, 2010.

[21] J.B. Hiriart-Urruty, C. Lemaréchal, Convex Analysis and Minimization Algorithms I, Springer, Berlin, 1993.

[22] J.P. Aubin, H. Frankowska, Set-Valued Analysis, Birkhäuser, Boston, 1990.

[23] P.A. Absil, R. Mahony, R. Sepulchre, Optimization Algorithms on Matrix Manifolds, Princeton University Press, Princeton, New Jersey, 2008.

[24] J. Jost, Riemannian Geometry and Geometric Analysis, Springer, Berlin, 2008.

[25] J.M. Lee, Introduction to Riemannian Manifolds, 2nd ed. Springer, New York, 2018.

[26] S. Lovett, Differential Geometry of Manifolds, CRC Press, 2019.

[27] T. Rapcsák, Smooth Nonlinear Optimization in $\mathbb{R}^{n}$. Kluwer Academic Publishers, Dordrecht, 1997.

[28] C. Udriste, Convex Functions and Optimization Methods on Riemannian Manifolds, Kluwer Academic Publishers, Dordrecht, 1994.

[29] T. Antczak, N. Abdulaleem, Optimality conditions for $E$-differentiable vector optimization problems with the multiple interval-valued objective function, J. Ind. Manag. Optim. 13 (2019), 1-19.

[30] A.A. Megahed, H.G. Gomma, E. A. Youness, A. H. El-Banna, Optimality conditions of $E$-convex programming for an $E$-differentiable function, J. Inequal. Appl. 2013 (2013), 1-11.

[31] G.R. Piao, L.G. Jiao, D.S. Kim, Optimality and mixed duality in multiobjective $E$-convex programming, J. Inequal. Appl. 2015 (2015) 1-13.

[32] E. A. Youness, $E$-convex sets, $E$-convex functions, and $E$-convex programming, J. Optim. Theory Appl. 102 (1999), 439-450.

[33] X. M. Yang, On $E$-convex sets, $E$-convex functions and $E$-convex programming, J. Optim. Theory Appl. 109 (2001), 699-704.

[34] R.T. Rockafellar, Convex Analysis, Princeton Math. Ser., Vol. 28, Princeton University Press, Princeton, New Jersey, 1970.

[35] M.A. Goberna, M.A. Lopéz, Linear Semi-Infinite Optimization, Wiley, Chichester, 1998. 
[36] M.S. Bazaraa, H.D. Sherali, C.M. Shetty, Nonlinear Programming: Theory and Algorithms, 3rd ed., WileyInterscience, New Jersey, 2006.

[37] B. Mond, T.Weir, Generalized concavity and duality. In: S. Schaible, W.T. Ziemba, (eds.) Generalized Concavity in Optimization and Economics, pp. 263-279, Academic Press, New York, 1981.

[38] P. Wolfe, A duality theorem for nonlinear programming, Quart. Appl. Math. 19 (1961), 239-244. 